\title{
Nonlinearities in industrial motion stages - detection and classification
}

Citation for published version (APA):

Rijlaarsdam, D. J., Loon, van, S. J. L. M., Nuij, P. W. J. M., \& Steinbuch, M. (2010). Nonlinearities in industrial motion stages - detection and classification. In Proceedings of the 2010 American Control Conference (ACC 2010), 30 June - 2 July 2010, Baltimore, Maryland, USA (pp. 6644-6649). Institute of Electrical and Electronics Engineers. https://doi.org/10.1109/ACC.2010.5531368

DOI:

10.1109/ACC. 2010.5531368

Document status and date:

Published: 01/01/2010

Document Version:

Accepted manuscript including changes made at the peer-review stage

Please check the document version of this publication:

- A submitted manuscript is the version of the article upon submission and before peer-review. There can be important differences between the submitted version and the official published version of record. People interested in the research are advised to contact the author for the final version of the publication, or visit the $\mathrm{DOI}$ to the publisher's website.

- The final author version and the galley proof are versions of the publication after peer review.

- The final published version features the final layout of the paper including the volume, issue and page numbers.

Link to publication

\section{General rights}

Copyright and moral rights for the publications made accessible in the public portal are retained by the authors and/or other copyright owners and it is a condition of accessing publications that users recognise and abide by the legal requirements associated with these rights.

- Users may download and print one copy of any publication from the public portal for the purpose of private study or research.

- You may not further distribute the material or use it for any profit-making activity or commercial gain

- You may freely distribute the URL identifying the publication in the public portal.

If the publication is distributed under the terms of Article 25fa of the Dutch Copyright Act, indicated by the "Taverne" license above, please follow below link for the End User Agreement:

www.tue.nl/taverne

Take down policy

If you believe that this document breaches copyright please contact us at:

openaccess@tue.nl

providing details and we will investigate your claim. 


\title{
Nonlinearities in Industrial Motion Stages - Detection and Classification
}

\author{
David Rijlaarsdam, Bas van Loon, Pieter Nuij and Maarten Steinbuch
}

\begin{abstract}
Detection and classification of nonlinearities in motion systems becomes of increasing importance with high demands on (closed loop) performance. In this paper two methods are compared that aim to measure both the linearized dynamics and the influence of nonlinearities. First, a broadband signal is used to measure a linear approximation of the systems dynamics. This method uses multisine signals with identical amplitude spectrum, but randomly distributed phases. Averaging over multiple periodic responses to the same signal and over multiple realizations of the random phase multisine allows the computation of the level of nonlinearities and external disturbances separately. This yields both a linear approximation of the systems dynamics and the amount of nonlinear 'disturbance' as a function of frequency. Second, single sine based measurements are used to measure the Higher Order Sinusoidal Input Describing Functions (HOSIDF) of the system under test. HOSIDFs describe the response of the system by describing not only the 'direct' response (gain and phase shift) of the system at the input frequency, but by describing the response at higher harmonics of the input frequency as well. This yields a quantitative measure of the power generated by nonlinearities at harmonics of the input frequency as a function of this frequency and the signal amplitude. In the paper these methods are utilized to acquire a non-parametric model for an industrial high precision stage. The effects of and sources for nonlinear influences are discussed for this particular case as well.
\end{abstract}

\section{INTRODUCTION}

In system identification, linearity is often assumed a priori. If nonlinear influences are small, such assumptions may be justified. However, the extend to which nonlinearities play a role in the system dynamics (and thus in possible control) should be assessed when performing system identification. This paper addresses two methods that combine the measurement of the linearized system dynamics with tools to detect the level and type of nonlinearities. In [10] an overview of methods that allow the detection of nonlinear behaviour is provided. After the introduction of the industrial application tested in this paper, a method based on the results in [7], [8], [9] is presented, which uses multisine signals to compute a Best Linear Approximation (BLA) of the systems dynamics and detect and classify possible nonlinearities. The second method is based on [4], [5] and measures the Higher Order Sinusoidal Input Describing Functions (HOSIDFs), relating the magnitude and phase of the harmonics in the output

This work is carried out as part of the Condor project, a project under the supervision of the Embedded Systems Institute (ESI) and with FEI company as the industrial partner. This project is partially supported by the Dutch Ministry of Economic Affairs under the BSIK program.

D.J. Rijlaarsdam, S.J.L.M. Van Loon, P.W.J.M. Nuij and M. Steinbuch are with the Departement of Mechanical Engineering of the Eindhoven University of Technology, Den Dolech 2, 5612 AZ Eindhoven, d.j.rijlaarsdametue.nl signal to a sinusoidal excitation. Finally, a comparison of both methods and views on future research are presented.

Note that multisine method provides the best linear approximation in a stochastic sense for the class of Gaussian excitation signals. Part of the result of the second method is a Linear Approximation (LA) of the systems dynamics as well. However, this LA does not incorporate possible frequency mixing phenomena Therefore, the obtained linearization is not necessarily the best or optimal one for the class of sinusoidal input signals. This difference also becomes clear from the analysis of both methods that follows in the sequel.

\section{MEASUREMENT SET-UP}

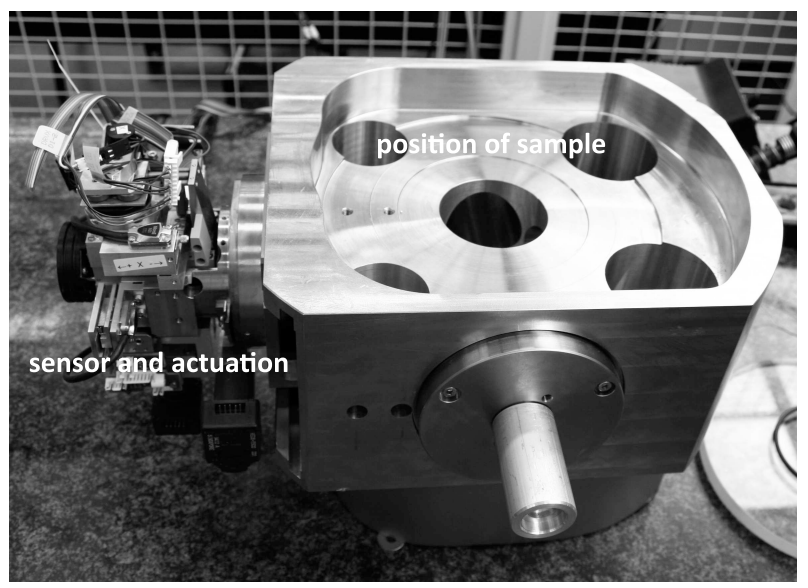

Fig. 1. System under test: an industrial high precision stage.

The system under test in this study is an industrial high precision stage (Figure 1). At the high resolution and reproducibility that is required in this case, nonlinear effects start to effect the dynamics of the system to such an extend that standard measurement techniques are no longer sufficient. This motivated the measurements presented in this paper. The stage is a SISO system with the motor voltage as the input and the position of the stage as its output. The system is excited and the response is measured using a SigLab 2042 dynamic signal analyzer providing $90[d B]$ aliasing protection and synchronized measurement channels. To measure the response of the system an industrial measurement system is used that allows high resolution position measurements over the entire stroke of the set-up.

\section{NONLINEAR DYNAMICS IN THE FREQUENCY DOMAIN}

The discussion in this paper is limited to the class of systems consisting of all stable, causal, time invariant, nonlinear 
systems which have a harmonic response to a harmonic input signal (see also [6]). A system is said to have a harmonic response to a sinusoidal input frequency if its response contains only spectral components $n \omega_{0}(n \in \mathbb{N})$, that are harmonically related to the input frequency $\omega_{0}$. In this paper, attention is focussed on this property which is exploited in both measurement strategies.

In [3] the author presents a way of systematically detecting nonlinearities that provides indications with respect to possible model choices. This method is based on one of the fastest ways to detect the presence of nonlinearities, which is the estimation of the frequency response function for different levels (power) of the excitation signal. Using band limited white noise with three different root mean square (rms) values, a preliminary experiment was performed. The results are depicted in Figure 2 and clearly indicate nonlinear behaviour.

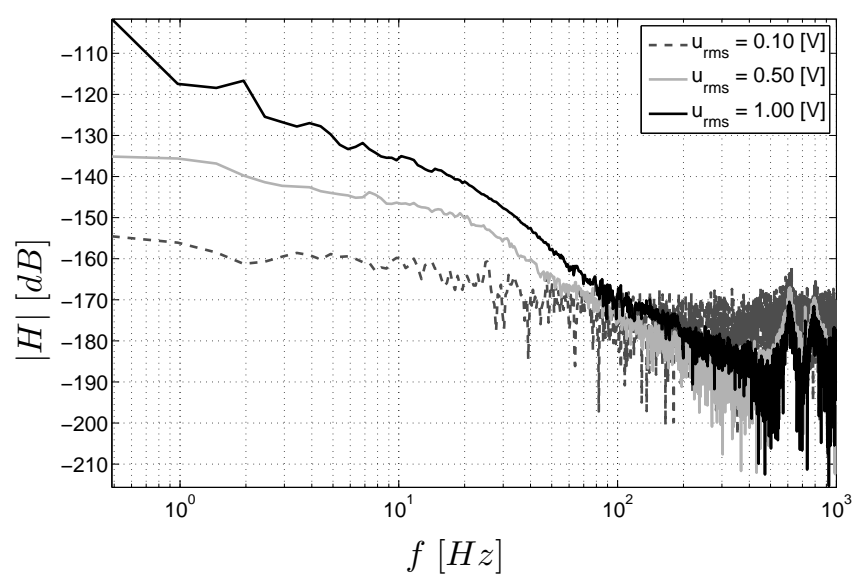

Fig. 2. Best linear approximation measured using band limited white noise input signals with different power.

\section{BROADBAND EXCITATION}

Two types of excitation signals are investigated in relation to the detection of nonlinear effects. This section deals with broadband excitations, while the next section provides an analysis based on single sine excitations. In the following the broadband excitation signals used in this paper are defined and the signal processing and analysis required to detect nonlinear effects are introduced. Finally, these methods are used to analyze experimental data.

A variety of broadband excitation signals exists, for example (white) noise and multisine excitations [7]. The latter consists of a sum of sinusoidal signals and is used in this paper. Multisine signals allow for leakage free measurements as opposed to (standard) noise excited measurements, where windowing is required to counteract the leakage. Furthermore, when the multisine is correctly selected [1], [8], [9], it is possible to identify the variances on the measured linearized dynamics due to stochastic distortions and nonlinear effects separately. Finally, it is possible to detect the type of nonlinearity in this way as well.

\section{Excitation Signal}

A multisine signal $u^{\mathfrak{b}}(t)$ is defined as a signal consisting of $N$ harmonic components with frequency $f_{n}^{\mathfrak{b}}[\mathrm{Hz}]$, amplitude $a_{n}^{\mathfrak{b}}$ and phase shift $\varphi_{n}^{\mathfrak{b}}$ :

$$
u^{\mathfrak{b}}(t)=\sum_{n=1}^{N} a_{n}^{\mathfrak{b}} \sin \left(2 \pi f_{n}^{\mathfrak{b}} t+\varphi_{n}^{\mathfrak{b}}\right),
$$

where ${ }^{\mathfrak{b}}$ denotes results and parameters related to broadband excitations. This type of excitation signal is fully defined by its harmonic content $f_{n}^{\mathfrak{b}} \in \mathbb{F}^{\mathfrak{b}} \subset \mathbb{R}_{\succ 0}$, it's amplitude spectrum $a_{n}^{\mathfrak{b}} \in \mathbb{A}^{\mathfrak{b}} \subset \mathbb{R}_{\succ 0}$ and the related phases $\varphi_{n}^{\mathfrak{b}} \in$ $\Phi^{\mathfrak{b}} \subset[-\pi \pi)$.

Next, consider a subset of the signals defined in Eq. (1), which consists solely of odd harmonics of a certain base frequency $f_{0}$ and randomly chosen phases. This signal type is referred to as the random odd multisine [9] and is defined rigorously below.

Definition 1 (Random odd multisine): The $\mathrm{m}^{\text {th }}$ realization of a random odd multisine is defined as:

$$
u^{[m], \mathfrak{b}}(t)=\sum_{n=1}^{N} a_{n}^{\mathfrak{b}} \sin \left(2 \pi f_{n}^{\mathfrak{b}} t+\varphi_{n}^{[m], \mathfrak{b}}\right),
$$

with $a_{n}^{\mathfrak{b}}$ possibly different for various frequencies, but constant over different realizations. The phases $\varphi_{n}^{[m], \mathfrak{b}} \in \Phi^{[m], \mathfrak{b}}$ are randomly selected from the interval $[-\pi \pi)$ for each realization and the odd harmonic frequency lines $f_{n}^{\mathfrak{b}} \in \mathbb{F}_{o}^{\mathfrak{b}}=$ $\left\{(2 k-1) f_{0}^{\mathfrak{b}} \mid k \in \mathbb{N}_{1}, f_{0} \in \mathbb{R}_{\succ 0}\right\}$ are selected identically for all realizations. The random odd multisine is particularly useful for detection of nonlinear effects when a frequency line is removed approximately every 5 odd frequency lines [8]. For convenience the same lines are removed for all realizations.

A more detailed analysis of the properties of these signals and the signal processing is presented in the next paragraph.

\section{Signal Processing and Analysis}

When using multisines as defined in Definition 1, there are two ways to detect nonlinearities in the spectral representation of the measured response. First of all, energy may appear on non-excited lines in the output spectrum. Secondly, a variance, larger than is to be expected based on stochastic distortions, is observed on the measured output spectrum, using different realizations of the multisine.

For the first detection method, a single measurement suffices. This method allows for the classification of the nonlinear effects (odd or even) since energy may appear on non-excited odd lines, on even lines or on both [8]. However, it requires an extrapolation of the effects observed at nonexcited lines to those at excited lines. Furthermore, this method does not provide a distinction between the variance of the output spectrum due to noise and the variance due to nonlinearities. To accomplish this, multiple experiments are required with different realizations of the multisine signal [1]. In this paper these detection methods are combined to both quantify the extend of nonlinear effects and classify them. 
Consider an experiment with $M$ realizations of the multisine signal defined in Definition 1. Furthermore, $P$ periods of the input signal $u(t)^{[m], \mathfrak{b}}$ and output signal $y(t)^{[m], \mathfrak{b}}$ are measured. This yields $P \times M$ periodic responses and the same number of estimates of discrete Fourier spectra of the output $Y(\omega)^{[m]}$. The superscript ${ }^{\mathfrak{b}}$ is omitted for the remainder of this section, to increase readability. Averaging over multiple periods of the same realization yields the variance on the average spectrum measured with this particular realization. This variance will consist of stochastic distortions (noise), but does not yield nonlinear effects since these occur in the same way in each of the measured periodic responses to one particular realization.

$$
\begin{aligned}
\bar{Y}^{[m]} & =\frac{1}{P} \sum_{p=1}^{P} Y_{p}^{[m]} \\
\sigma_{\bar{Y}, \text { noise }}^{2^{[m]}} & =\frac{1}{P(P-1)} \sum_{p=1}^{P}\left(Y_{p}^{[m]}-\bar{Y}^{[m]}\right)^{2}
\end{aligned}
$$

The average spectra $\bar{Y}^{[m]}$ consist both of the excited lines and a possible response on non-excited lines. Excited lines are the frequency lines that are present in the spectrum of the input signal. Therefore, the analysis splits into two parts. First, the response on the excited lines will be analyzed and second, the computation of the response measured on non-excited lines will be discussed. For the frequency points where the system was originally excited, the part of the output spectrum that is generated by the Best Linear Approximation (BLA) of the system and the corresponding variance are calculated.

$$
\begin{aligned}
Y_{B L A} & =\frac{1}{M} \sum_{m=1}^{M}\left[\frac{U^{[m]}}{\left|U^{[m]}\right|}\right]^{-1} \bar{Y}^{[m]} \\
M \sigma_{Y_{B L A}, N L}^{2} & +\sigma_{Y_{B L A}, \text { noise }}^{2} \\
& =\frac{1}{M-1} \sum_{m=1}^{M}\left(\bar{Y}^{[m]}-Y_{B L A}\right)^{2}
\end{aligned}
$$

where $\left(U^{[m]} /\left|U^{[m]}\right|\right)^{-1}$ in equation (5) corrects the phases of the output spectra with respect to the corresponding input spectrum, prior to averaging. Furthermore, $\sigma_{Y_{B L A}, \text { noise }}^{2}$ is the average of the variance induced by stochastic distortions on the individual measurements of the different realization as computed from Eq. (4). The variance on the averaged spectrum over all realization which is due to nonlinear influences is denoted by $\sigma_{Y_{B L A}, N L}^{2}$. This variance is calculated by analyzing the variance over different realizations since the nonlinearities are excited differently for different realizations of the multisine. Note that the calculations above may be performed on the Frequency Response Function (FRF) as well as the measured output spectra. In case of stochastic analysis of the FRF, no phase correction is required.

The response at non-excited lines is obtained by calculating the variance of the power in the output spectra, measured at the non-exited lines. The spectrum has random phase at these lines, but calculating the variance yields the power that occurs at these frequencies. A distinction is made between energy occurring at odd and even lines for classification of the nonlinearities:

$$
\mathcal{P}_{Y}\left(\ell_{o / e}\right)=\frac{1}{M-1} \sum_{m=1}^{M}\left(\varepsilon-\bar{Y}^{[m]}\left(\ell_{o / e}\right)\right)^{2},
$$

where $\ell_{o / e}$ denotes the odd and even non-excited frequency lines respectively. The average value $\varepsilon$ is small, since the phases of $\bar{Y}^{[m]}\left(\ell_{e / o}\right)$ are randomly distributed in the interval $[-\pi \pi)$ and the average therefore tends to zero.

Summarizing, by conducting measurements with $P$ periods of $M$ realizations of the random multisine (Definition 1), the Best Linear Approximation (BLA) of the Frequency Response Function or of the part of the output spectrum generated by this BLA, is obtained using Eq. (5). Furthermore, the variance on this BLA due to stochastic disturbances is computed according to Eq. (4). The influence of nonlinearities can be computed as a variance of the output spectrum or FRF by Eq. (6). Finally, an estimate of nonlinear effects as well as a classification of these effects is obtained from Eq. (7).

\section{Experimental Results}

The method described in the previous section is applied to measure the BLA and the level of nonlinearities in the industrial high precision stage described in Section II. Measurements are performed using a SigLab measurement system with a measurement frequency of $f_{s}=2560[\mathrm{~Hz}]$ and a block length of $N_{\text {block }}=8192$ measurement points. The multisine signal is defined according to Definition 1, with $f_{0}^{\mathfrak{b}}=\frac{f_{s}}{N_{\text {block }}}=0.3125[\mathrm{~Hz}]$. Sufficient waiting time is allowed to assure that transient phenomena have damped out, avoiding leakage phenomena (no windowing is applied).

$M=10$ realizations of the odd random multisine have been generated and the response has been measured for $P=$ 10 periods (approx. 32 seconds). Furthermore, this experiment is repeated for 20 different rms values of the multisines, logarithmically scaled between $0.3[V]$ and $5.0[V]$. This leads to a total of $20 \times P=200$ input-output measurements consisting of 10 sequential periods each. After applying the above described signal processing and analysis to each of the measurement sets, a typical output spectrum is depicted in Figure 3.

Figure 3 shows that nonlinear effects have an average level that is $10[d B]$ lower than the power generated in the output spectrum by the BLA of the system. These nonlinearities have both an odd and even nature, but the odd components dominate since they appear at a level that is almost $20[d B]$ higher than that of the even nonlinearities. Furthermore, it becomes clear that the variation due to nonlinearities is of the same order of magnitude as the odd nonlinearities that are detected. Finally the variation due to process and / or measurement noise is almost $30[d B]$ lower than than the variation due to nonlinear effects.

Figure 4 depicts the BLA of the system as a function of both the rms value and frequency of the input. Figure 5 shows three cross section of Figure 4 at high, medium and 


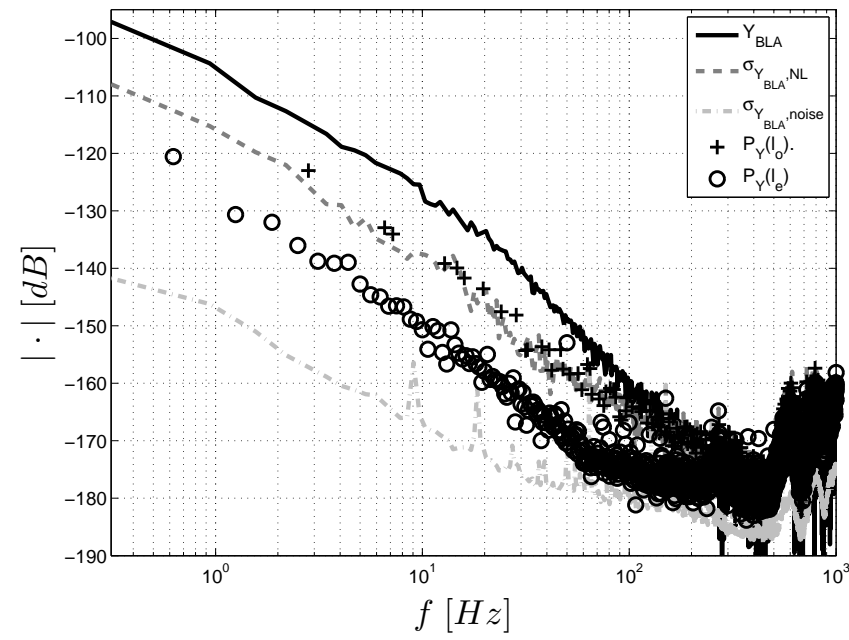

Fig. 3. Output spectrum of a typical multisine experiment, showing the effects of nonlinearities $\left(\sigma_{Y_{B L A}, N L}\right)$ and noise $\left(\sigma_{Y_{B L A}, n o i s e}\right)$ as variances on the best linear approximation of the spectrum $\left(u_{r m s}=\right.$ $3.72[V])$

low input power. The difference in the computed BLA and corresponding variation emphasizes the dependency on input power as well. From Figure 4 and 5 it becomes clear that for high input power the response of the system becomes more linear. This becomes clear from the fact that the both the gradient $\partial H / \partial u_{r m s}$ and the value of the variation due to nonlinear influences decreases relative to the BLA of the gain of the system decreases for increasing $u_{r m s}$.

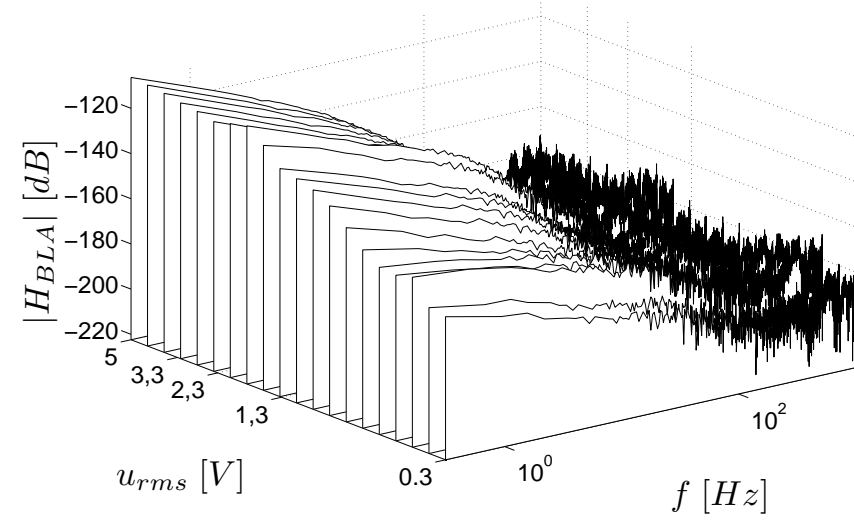

Fig. 4. Best linear approximation $H_{B L A}$, measured and calculated using the broadband excitation approach, as a function of input amplitude and frequency.

\section{SINGLE SINE EXCITATION}

Apart from broadband excitations, single sine based excitations signals provide means to detect nonlinear effects as well. Rather than using the variance on measured output spectra, this method exploits the fact that nonlinearities cause

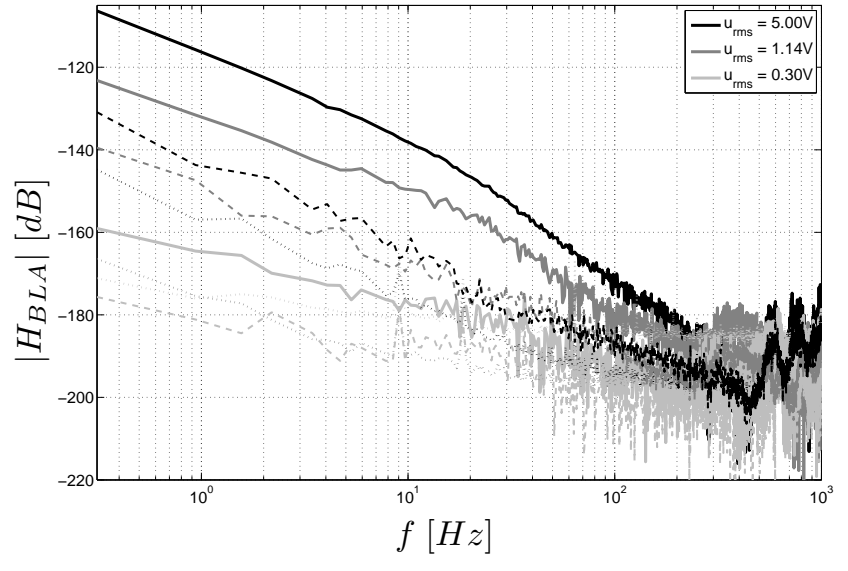

Fig. 5. Best linear approximation, measured and calculated using the broadband excitation approach, for various values of the power of the input signal (rms value). Measured $H_{B L A}(-)$, standard deviation $\left(\sigma_{H_{B L A}, N L}\right)$ due to nonlinear effects $(--)$ and standard deviation $\left(\sigma_{H_{B L A}, \text { noise }}\right)$ due to measurement and / or process noise $(\cdot)$.

energy to appear in the output spectrum on frequency lines that are not present in - but harmonically related to - the input spectrum. This phenomenon was investigated in the previous section as well, when analyzing nonlinear influences using broadband signals. However, in this case the energy observed on non-excited lines was due to a mixture of all (or a subset) of the excited frequencies. This section will focus in particular on describing how this energy is generated at non-excited lines as a function of the (single) frequency and amplitude of the input signal.

The principle used to visualize and measure the influence of nonlinearities in the frequency domain is that of the Higher Order Sinusoidal Input Describing Functions (HOSIDF). This section starts with the definition of the HOSIDFs and the excitation signal used. It continues with a description of the analysis and processing of the measured signals and the computation of the HOSIDFs. Finally, experimental results are presented that illustrate this method in an industrial application.

\section{Higher Order Sinusoidal Input Describing Functions}

A variety of describing functions exist of which the Sinusoidal Input Describing Function (SIDF) is a well known example [2]. The SIDF describes the response of a nonlinear system to a sinusoidal input signal by only considering the response at the input frequency. For linear systems the SIDF therefore reduces to the well known frequency response function. For nonlinear systems the SIDF also considers only the 'direct' response (gain and phase shift) at the input frequency and assumes that no harmonic content is generated at frequencies other than the input frequencies.

In order to model systems with levels or types of nonlinearities that do not satisfy this assumption the concept of Higher Order Sinusoidal Input Describing Functions (HOSIDF) was developed [4], [5]. HOSIDFs describe the response of the system by describing not only the response 
of the system at the input frequency, but by describing the response at harmonics of the input frequency, generated by nonlinearities, as well. In order to accomplish this, the concept of the harmonic generator is introduced. This virtual nonlinear element receives a single harmonic sine wave at its input and generates an infinite number of output signals with equal amplitude but frequencies that are harmonically related to the spectral content of the input signal. More specifically, these output signals have frequencies and phases that are exact multiples $k \omega_{0}, k \varphi_{0}, k \in \mathbb{N}$ of the frequency $\omega_{0}$ and phase $\varphi_{0}$ of the input signal. For each of these channels the response is described separately by the corresponding HOSIDF $\mathcal{H}_{k}\left(a^{\mathfrak{s}}, f_{0}^{\mathfrak{s}}\right)$, which may be a function of both the input amplitude and frequency. The systems output is a sum of all $k$ responses.

The $\mathrm{k}^{\text {th }}$ order HOSIDF is defined as:

$$
\begin{aligned}
\left|\mathcal{H}_{k}\left(a^{\mathfrak{s}}, f_{0}^{\mathfrak{s}}\right)\right| & =\frac{\left|Y\left(k f_{0}^{\mathfrak{s}}\right)\right|}{\left|U\left(f_{0}^{\mathfrak{s}}\right)\right|} \\
\angle \mathcal{H}_{k}\left(a^{\mathfrak{s}}, f_{0}^{\mathfrak{s}}\right) & =\angle Y\left(k f_{0}^{\mathfrak{s}}\right)-k \angle U\left(f_{0}^{\mathfrak{s}}\right),
\end{aligned}
$$

where ${ }^{\mathfrak{s}}$ denotes results and parameters related to single sine excitation signals, $Y\left(k f_{0}^{\mathfrak{s}}\right) \in \mathbb{C}$ is the output spectrum at the $\mathrm{k}^{\text {th }}$ harmonic frequency line and $U\left(f_{0}^{\mathfrak{s}}\right) \in \mathbb{C}$ the spectral content of the input signal at its fundamental frequency $f_{0}^{\mathfrak{s}} \in \mathbb{R}_{\succ 0}$. HOSIDFs are generally a function of both the input frequency $f_{0}^{\mathfrak{s}}$ and amplitude $a^{\mathfrak{s}} \in \mathbb{R}_{\succ 0}$, where the input signal is defined as:

$$
u^{\mathfrak{s}}(t)=a^{\mathfrak{s}} \sin \left(2 \pi f_{0}^{\mathfrak{s}} t\right) .
$$

The aim of this part of the paper is to measure and describe the HOSIDFs by conducting a series of experiments with varying input frequency and amplitude. The related signal processing and analysis is discussed in the next paragraph.

\section{Signal Processing and Analysis}

In order to measure a single HOSIDF $\mathcal{H}_{k}\left(a^{\mathfrak{s}}, f_{0}^{\mathfrak{s}}\right)$ for a given frequency / amplitude combination, an input signal as defined in Eq. (10) is applied and the spectrum of the response is computed using standard DFT algorithms and Eqs. (8) - (9). By repeating the experiment $R$ times the average and variance on the measured HOSIDF can be evaluated according to:

$$
\begin{aligned}
\overline{\mathcal{H}}_{k}\left(a^{\mathfrak{s}}, f_{0}^{\mathfrak{s}}\right) & =\frac{1}{R} \sum_{r=1}^{R} \mathcal{H}_{k}^{[r]}\left(a^{\mathfrak{s}}, f_{0}^{\mathfrak{s}}\right) \\
\varsigma_{\overline{\mathcal{H}}_{k}}^{2}\left(a^{\mathfrak{s}}, f_{0}^{\mathfrak{s}}\right) & =\frac{1}{R(R-1)} \sum_{r=1}^{R}\left(\mathcal{H}_{k}^{[r]}(\cdot)-\overline{\mathcal{H}}_{k}(\cdot)\right)^{2}
\end{aligned}
$$

By repeating the experiments for different amplitudes and frequencies the HOSIDFs are obtained. In the sequel, experimental results are presented that illustrate these results.

\section{Experimental Results}

Measurements have been performed by exciting the system introduced in Section II, with frequencies ranging from $5[\mathrm{~Hz}]$ to $300[\mathrm{~Hz}]$ in steps of $5[\mathrm{~Hz}]$. Furthermore, each response has been measured for a variety of input signal powers $u_{r m s}=a^{\mathfrak{s}} / \sqrt{2}$, ranging from $0.07[V]$ to $1.41[V]$ (logarithmically spaced). For each frequencyamplitude combination, 10 experiments are performed which allows calculation of the mean and variance of the measured value. All measurements have been performed using a SigLab measurement system with a measurement frequency of $5120[\mathrm{~Hz}]$ and a block length of 2048 points. This results in leakage free measurements since sufficient waiting time is allowed for the transient response to damp out.

A typical series of HOSIDFs is depicted in Figure 6. Since the even HOSIDFs are very low in most cases, only the odd HOSIDFs are considered in this paper. From Figure 6 it becomes clear that the system becomes more linear for increasing value of $u_{r m s}$. This can be observed from the combination of a decreasing gradient $\partial \mathcal{H}_{1} / \partial u_{r m s}$ and a decreasing value of $\mathcal{H}_{i} \forall i \in N_{\succ 1}$ for all measured frequencies.

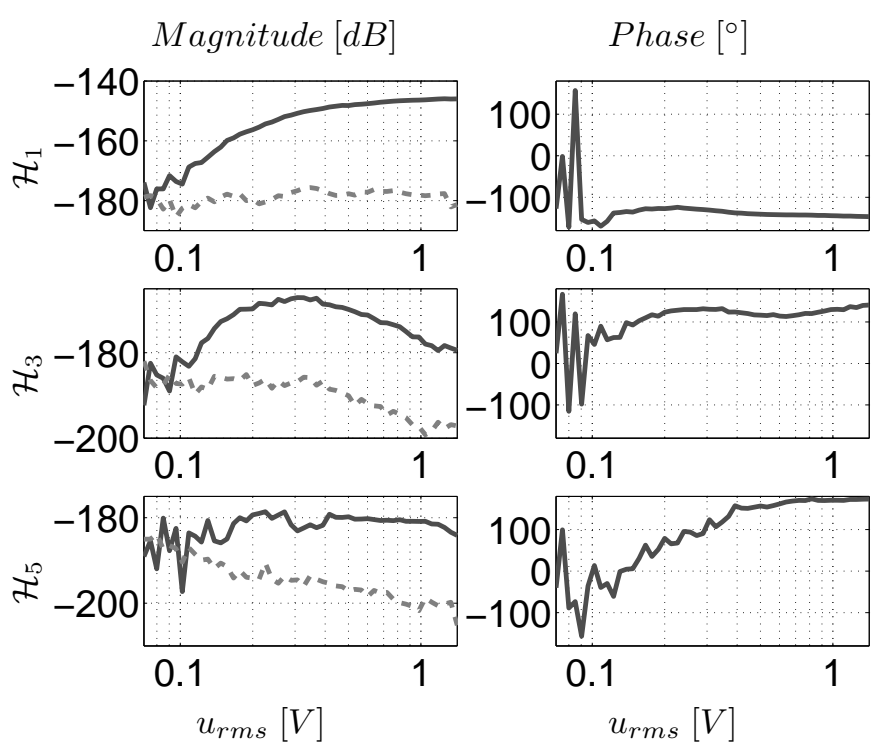

Fig. 6. HOSIDFs at $20[\mathrm{~Hz}]$ calculated from 10 experiment per amplitude. Mean value $\left(\overline{\mathcal{H}}_{k}\right)$ of the $\operatorname{HOSIDF}(-)$ and standard deviation $\varsigma_{\mathcal{H}_{k}}(--)$.

In Figure $7\left|\mathcal{H}_{1}\right|$ is depicted as a function of input power and frequency. The results depicted in this figure may be compared to those depicted in Figure 4.

\section{COMPARISON}

The methods discussed in this paper both result in a linear approximation of the systems dynamics. In general these results will differ, since the multisine based method results in a statistical best linear approximation, while the HOSIDFs provide a linear approximation (LA) that does not incorporate frequency mixing. Furthermore, the LA generally depends on the signal type that is used for identification and Gaussian multisine signals have a different amplitude probability density function (pdf) than single sine signals, which in general leads to different linearizations as well.

The methods also differ in measurement time and computational costs. The single sine measurement required 30000 measurements versus 200 measurements using a multisine. 


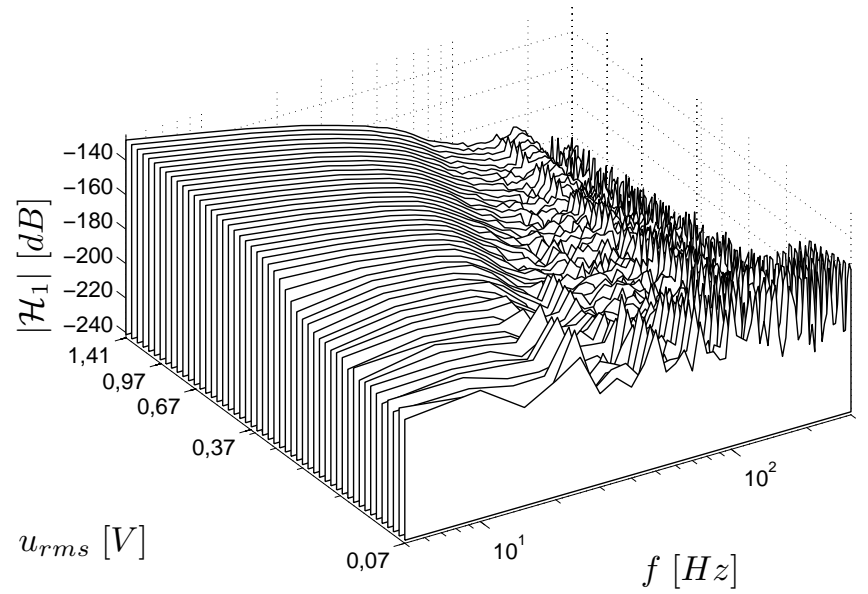

Fig. 7. Linear approximation $\left(\overline{\mathcal{H}}_{1}\right)$, measured and calculated using the single sine approach, as a function of input amplitude and frequency.

Therefore, the measurement using multisine signals might appear more appealing at first sight. However, although the nature and level of nonlinearities may be estimated using this method, it lacks detailed information provided by the HOSIDFs, about how frequency content (phase and amplitude) is generated as a function of input frequency and amplitude. Furthermore, the time argument depends on the ratio between the required effective measurement time and time constants of the system.

The LAs that are computed using both methods are depicted in Figure 8. As becomes clear the linear approximations are similar for high excitation power, except for a high frequency roll-off (single sine measurement). The main reason for this similarity at high excitation power, is that the system behaves close to linear with respect to excitation signal type, pdf and the generation of harmonic content. However, both methods indicate a dependency of the BLA on the excitation level, which does indicate nonlinear behaviour with respect to excitation power.

\section{CONCLUSION \& FUTURE RESEARCH}

The methods considered in this paper are both shown to be effective in measuring a linearization of the system dynamics. The measurement time and computational cost differ and depending on the time scale of the system under test, long transient times might lead to a preference for the multisine based method. However, HOSDFs provide more detailed information about the nonlinearities present. Apart from detailed magnitude information about nonlinear influences, the HOSIDF also include phase information. This information is not (directly) available from the multisine based method. Both methods indicate that the odd nature of the nonlinearity is dominant over the even nonlinearities and that the influence of nonlinearities decreases with increasing excitation level. It is therefore expected that the main source of nonlinear behaviour is friction in this case.
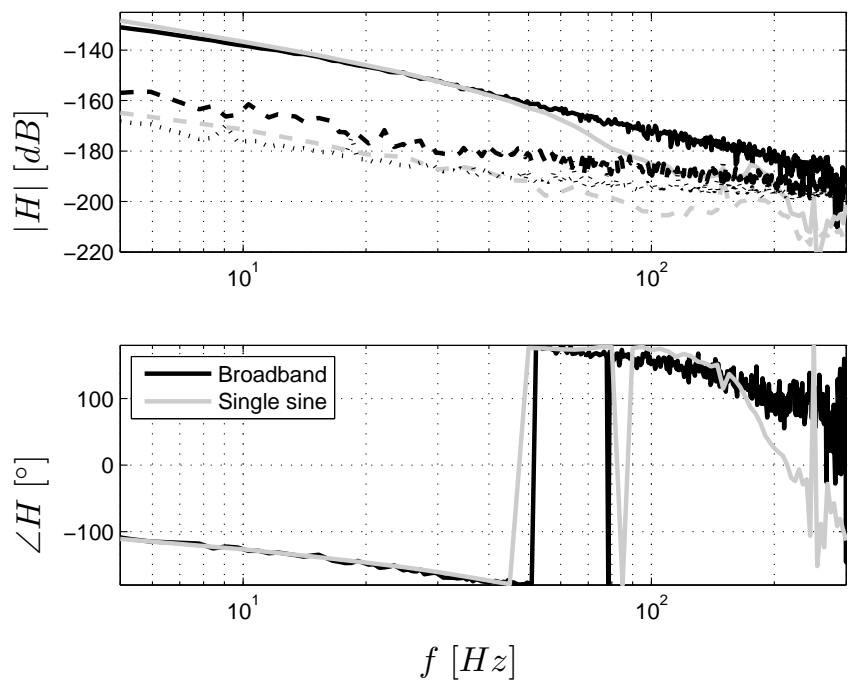

Fig. 8. Comparison of the best linear approximation (for high excitation amplitude), calculated using the broadband excitation approach $\left(u_{r m s}=\right.$ $5.0[V]$ and the single sine excitation approach $\left.\left(u_{r m s}=1.41[V]\right)\right)$. Top figure: (black) results obtained with broadband excitations (-) magnitude of $H_{B L A},(--) \sigma_{H_{B L A}, N L},(\cdot) \sigma_{H_{B L A}, \text { noise }}$ and (grey) single sine excitations (-) magnitude of $\overline{\mathcal{H}}_{1},(--) \varsigma_{\mathcal{H}_{1}}$.

Future research will focus on further comparison of these methods. Finally, for industrial purposes, the multisine signal is currently more appealing than the HOSIDFs, since the level of nonlinear distortion may be used in robust control. The usage of HOSIDFs in control and particularly in nonlinear controller synthesis will be addressed in future research.

\section{REFERENCES}

[1] D'haene, T., R. Pintelon, J. Schoukens, E. v. Gheem (2005). Variance analysis of frequency response funtion measurements using periodic excitations, Proc. ISMA2002, vol. 2, pp. 1241-1256.

[2] Gelb, A., Velde, W.E. van er (1968). Multiple input describing functions and nonlinear system design, McGraw Hill, New York.

[3] Lauwers, L., J. Schoukens, R. Pintelon, M. Enqvist (2008). Summary and comparing overview of techniques for the detection of nonlinear distortions, IEEE Trans. Instr. Meas., vol. 57, pp. 2257-2264.

[4] Nuij, P.W.J.M., O.H. Bosgra, M. Steinbuch (2006). Higher-order sinusoidal input describing functions for the analysis of non-linear systems with harmonic responses, Mechanical Systems and Signal Processing, vol. 20, pp. 1833-1904.

[5] Nuij, P.W.J.M., M. Steinbuch, O.H. Bosgra (2008). Measuring the higher order sinusoidal input describing functions of a non-linear plant operating in feedback, Control Engineering in Practice, vol. 16, pp. 101-113.

[6] Pavlov, A. (2004). The output regulation problem - convergent dynamics approach, $\mathrm{PhD}$ thesis, Eindhoven University Press, (ISBN 90-3862736-X).

[7] Pintelon, R., J. Schoukens (2001). System identification: a frequency domain approach, IEEE Press Piscataway, NJ, (ISBN 0-7803-6000-1).

[8] Pintelon, R., G. Vandersteen, L. De Locht, Y. Rolain, J. Schoukens (2004). Experimental characterization of operational amplifiers: a system identification approach, IEEE Trans. Meas. Instr., vol. 53, no. 3, pp. 854-867.

[9] Vanhoenacker, K., T. Dobrowiecki, J. Schoukens (2001). Design of multisine excitations to characterize the nonlinear distrotions during FRF measurements, IEEE Trans. Meas. Instr., vol. 50, no. 5, pp. 10971102.

[10] Vanhoenacker, K., J. Schoukens, J. Swevers, D. Vaes (2002). Summary and comparing overview of techniques for the detection of nonlinear distortions, Proc. ISMA2002, vol. 2, pp. 1241-1256. 